\title{
Contrast imaging techniques and renal dysfunction
}

\begin{abstract}
Iodinated contrast has been one of the most prescribed and used drugs in contrast imaging techniques and interventional procedures. However, some subjects may develop contrast-induced nephropathy (CIN) especially those in advanced chronic kidney disease (CKD). Diagnostic criterion is based on increasing $48 \mathrm{~h}$ creatinine after receiving iodine. Given the fact that there is no specific treatment for CIN, prevention should be considered. Uncountable actions should be taken, including: reducing the dose, using a low-osmolar substance, avoiding dehydration and other nephrotoxic drugs. Currently, prevention has been based on using saline solution. A recent paper showed that for patients with stage 3 and 4 of CKD sodium bicarbonate did not provided greater benefit when compared to a saline solution, as well as comparing acetylcysteine to placebo. Regarding to gadolinium, besides nephrotoxicity is irrelevant, there is a risk for developing Nephrogenic Systemic Fibrosis (NSF) which may occur in those with GFR $<60 \mathrm{~mL} / \mathrm{min}$, particularly appears at a GFR $<30 \mathrm{~mL} / \mathrm{min}$. Current gadolinium use guidelines are related to patients with stage 5 (GFR $<15 \mathrm{~mL}$ / min) according to which they should undergo hemodialysis after examination.
\end{abstract}

Volume 5 Issue I - 2018

\author{
Benedito Jorge Pereira,' Germana Alves de \\ Brito,' Danilo Euclides ${ }^{2}$ \\ 'AC Camargo Cancer Center, Brazil \\ ${ }^{2}$ University Federal of Sao Paulo, Brazil
}

Correspondence: Benedito Jorge Pereira,AC Camargo Cancer Center, Antonio Prudente Street 2II, São Paulo- SP, Brazil,Tel 55। |21895000,

Email benedito.pereira@accamargo.org.br

Received: December 26, 2017 | Published: January 25, 2018

Keywords: iodinated contrast, nephrotoxicity, gadolinium, nephrogenic systemic fibrosis, chronic kidney disease

Abbreviations: CIN, contrast-induced nephropathy; CI-AKI, contrast-induced aki; CKD, chronic kidney disease; Cr, creatinine; GFR, glomerular filtration ratio; NSF, nephrogenic systemic fibrosis; AMI, acute myocardial infarction; NSAIDs, nonsteroidal anti-inflammatory drugs; AKI, acute kidney injury; ATN, acute tubular necrosis; TIN, tubulointerstitial nephritis

\section{Introduction}

Iodinated contrast has been one of the most prescribed and used drugs in contrast imaging techniques and interventional procedures. Cancer hospitals frequently use it for diagnosing and staging diseases. Due to clinical complications, such as acute myocardial infarction (AMI) and angina, General hospitals use it for coronary angiography and angioplasty as well as tomography and angiography for those with stroke as a diagnosing and interventionist instrument.

A patient who will receive a contrast approach should be aware of its safety, as well as its risks and clinical benefits for assistance of diagnosed diseases. ${ }^{1}$ In this brief review, we are going to discuss how to diagnose and prevent complications related to contrast use such as contrast-induced nephropathy $(\mathrm{CIN})$ and nephrogenic systemic fibrosis (NSF) after using gadolinium.

\section{Discussion}

\section{Contrast-induced nephropathy}

Frequently, contrast-induced nephropathy (CIN) occurs in advanced stages of chronic kidney disease (CKD) which increases risk of needing a renal replacement therapy (RRT). ${ }^{2}$ Diagnostic criterion is based on increasing $48 \mathrm{~h}$ creatinine after receiving iodine. Renal biopsy is required only for excluding other causes of acute kidney injury (AKI). ${ }^{3}$ Given the fact that there is no specific treatment for CIN, prevention should be considered. Uncountable actions should be taken, including: reducing the dose $(<200 \mathrm{~mL})$, wait longer for repeating exams ( $>72$ hours), using a iso or hypoosmolar contrast, avoiding dehydration during the exam, stopping using nephrotoxic drugs such as nonsteroidal anti-inflammatory drugs (NSAIDs) and using periprocedural saline solution. ${ }^{4}$

Dehydration and glomerular filtration ratio (GFR) $<60 \mathrm{~mL} / \mathrm{min}$ are the most important risk factor of developing contrast-induced AKI (CI-AKI). However, others morbidities such as diabetes mellitus, cardiac insufficiency and reduction of effective plasma volume as in cirrhosis and in nephrotic syndrome are associated to a greater risk of CIN development. ${ }^{4-6}$ Besides knowing this frequent cause of AKI it is important in cases of emergency to rank individuals according to the risk of developing CI-AKI as showed in Table 1.

\section{Contrast nephropathy differential diagnosis}

Differential diagnosis includes renal atheroembolism, ischemic acute tubular necrosis (ATN), tubulointerstitial nephritis (TIN) and pre-renal AKI after contrast. In people who develops AKI after angiography renal atheroembolism it should be distinguished by presenting at least one of the following characteristics: presence of embolic lesions (digital ischemia) or livedo reticularis, transient eosinophilia or complement consumption, AKI that appears days after being exposed to contrast and long lasting with no recovery of renal function (or low recovery). ${ }^{7}$

\section{Contrast-induced acute kidney injury prevention}

Hydration with saline solution $0,9 \%$ is possibly the unique way to prevent CI-AKI. However, there are no guidelines regarding do "how much" and "how long". It is suggested to use $1 \mathrm{~mL} / \mathrm{kg} /$ hour of saline solution at least 6hours before the procedure. , $, 6,8^{\circ}$

Bicarbonate hydration protocol may be useful for emergencies especially for the circumstances there are not enough time for hydrating with saline solution. Still, bicarbonate was not more effective in preventing CIN in that cases which RRT was necessary or in cases of death prevention. ${ }^{8}$ Prophylaxis using bicarbonate solution predicts to dilute $150 \mathrm{mEq}$ of bicarbonate in $850 \mathrm{~mL}$ of glucose serum $5 \%$. It 
is recommended to use $3.0 \mathrm{~mL} / \mathrm{kg}$ anhour before and, for those who could not take the hydration or who were in emergency condition, $1.0 \mathrm{~mL} / \mathrm{kg}$ 6hours after the procedure. ${ }^{4,6}$

Table I Cl-AKI risk stratification ${ }^{3}$

\begin{tabular}{|c|c|c|c|c|}
\hline & $\begin{array}{l}\text { Risk } \\
\text { prediction }\end{array}$ & $\begin{array}{l}\text { Risk } \\
\text { score }\end{array}$ & $\begin{array}{l}\text { Cl-AKI } \\
\text { risk }\end{array}$ & $\begin{array}{l}\text { Hemodialysis } \\
\text { risk }\end{array}$ \\
\hline Hypotension & 5 & & & \\
\hline $\begin{array}{l}\text { Intra-aortic } \\
\text { balloon pump }\end{array}$ & 5 & $\leq 5$ & $7,5 \%$ & $0,04 \%$ \\
\hline $\begin{array}{l}\text { Congestive heart } \\
\text { failure }\end{array}$ & 5 & & & \\
\hline Age $>75$ anos & 4 & 10-Jun & $14 \%$ & $0,12 \%$ \\
\hline Anemia & 3 & & & \\
\hline Diabetes & 3 & 16-Nov & $26,1 \%$ & ।,09\% \\
\hline $\begin{array}{l}\text { Contrast-media } \\
\text { volume }\end{array}$ & $\mathrm{I} / \mathrm{I00 \textrm {ml }}$ & & & \\
\hline $\mathrm{SCr}>\mathrm{I}, 5 \mathrm{mg} / \mathrm{dl}$ & 4 & $\geq 16$ & $57,3 \%$ & $12,6 \%$ \\
\hline \multicolumn{5}{|l|}{ GFR } \\
\hline $40-60 \mathrm{~mL} / \mathrm{min}$ & 2 & & & \\
\hline $20-40 \mathrm{~mL} / \mathrm{min}$ & 4 & & & \\
\hline$<20 \mathrm{~mL} / \mathrm{min}$ & 6 & & & \\
\hline
\end{tabular}

Regarding to the contrast substance use, low-osmolar, nonionic substances are safer than high-osmolar ones. Yet, recent papers has not showed that non ionic substances are better than low-osmolar ones. ${ }^{6,9}$ Despite no confirmation of protection through acetylcysteine or bicarbonate hydration ${ }^{8}$ in those individuals with GFR $<60 \mathrm{~mL} / \mathrm{min}$ and in emergency cases these strategies could be considered but not without thinking about other approaches. It might be mentioned that there is no evidence of what should be the best prevention strategy. ${ }^{4,6,10}$ Acetylcysteine can be given orally $600 \mathrm{mg} 12 / 12$ hours or, as mentioned by some research $1200 \mathrm{mg}$ intravenously $12 / 12$ hours. ${ }^{11,12}$

Recent evidences do not support that statins and vitamin $\mathrm{C}$ prevent CIN from happening. ${ }^{2}$ In spite of that a randomized research that evaluated trimetazidine use of $35 \mathrm{mg} 12 / 12$ hours $48 \mathrm{~h}$ before the contrasted exams for those with GFR between 30 and $90 \mathrm{~mL} / \mathrm{min}$ to take isotonic saline solution $1 \mathrm{~mL} / \mathrm{kg} / \mathrm{h}$ and trimetazidine versus placebo observed a reduction of CIN incidence in the group that was treated with saline solution and trimetazidine. ${ }^{13}$ Table 2 shows some remarkable strategies for preventing CI-AKI.

\section{Gadolinium contrast}

Besides gadolinium has not been related to complications such as AKI for those with previous renal insufficiency, this paramagnetic contrast substance which is used for magnetic resonance exams may develop an incapacitating disease that is not well-known yet. It can progressively affect skin, joints and some organs such as lungs and heart. Its pathophysiological mechanism results from the release of gadolinium especially those from linear molecules from its gadolini- um-chelate complex that binds to body metals such as iron and zinc. Then, it can be accumulated in skin, joints and organs and may cause progressive tissue fibrosis. First symptoms are skin thickening and pruritus progressing rapidly to contracture and immobility for those who were affected. ${ }^{14,15}$ Among patients who have greater risk for developing Nephrogenic Systemic Fibrosis (NSF) are those with GFR $<60 \mathrm{~mL} / \mathrm{min}$ and mainly those with GFR $<30 \mathrm{~mL} / \mathrm{min}$. Table 3 shows risk factors for developing Nephrogenic Systemic Fibrosis (NSF).

Table 2 Cl-AKI prevention
To evaluate cost-effective of its benefits.
To evaluate eGFR.
To avoid furosemide 24 hours before and after the procedure.
If possible, to avoid nephrotoxic drugs combined with NSAIDs, aminoglycosides. Hydration with saline solution $0,9 \% \mathrm{ImL} / \mathrm{kg} / \mathrm{h}$ 6- I 2 hours before
the procedure and right before it.

Hydration with bicarbonate solution (I50mEq bicarbonate $/ 850 \mathrm{~mL}$ glucose serum $5 \%$ ) $3,0 \mathrm{~mL} / \mathrm{kg}$ an hour before the procedure and $1,0 \mathrm{~mL} / \mathrm{kg}$ 6hours after it for those who could not take it before and for those who were emergency cases.

To give iso or low-osmolar contrasts and non-ionic substances.

To give the lower possible volume of contrast $(<2 \mathrm{~mL} / \mathrm{kg})$.

To avoid oral hypoglycemic agents during the procedure (risk of hypoglycemia and acidosis in those who may develop AKI).

To recommend 72 hours between exams in cases it should be redone.

To give $\mathrm{n}$-acetylcysteine $600 \mathrm{mg} / 2 / 12 \mathrm{~h}$ before and after the procedure for those with renal dysfunction (there are controversies).

Table 3 Risk factor for developing nephrogenic systemic fibrosis (NSF)

Patients with stages 4 and 5 of chronic kidney disease (10-13\%).

Linear molecules gadolinium.

Gadodiamide (the most related to NSF), and there are some evidences for Gadopentetate and gadoversetamide.

Bigger doses and numerous expositions to the substance.

Erythropoietin use.

Serum iron supplementation.

Patients with vascular disease.

For those who are in dialysis therapy it is recommended to dialyze right after being exposed to contrast (gadobutrol - gadovist) and the two following days after being taking gadolinium (Table 4). Metformin may not be suspended when gadolinium will be used. Current recommendations regarding to gadolinium use shows that just those with end-stage renal disease should dialyze right after the procedure. ${ }^{16}$ 
Table 4 Gadolinium classification manual I

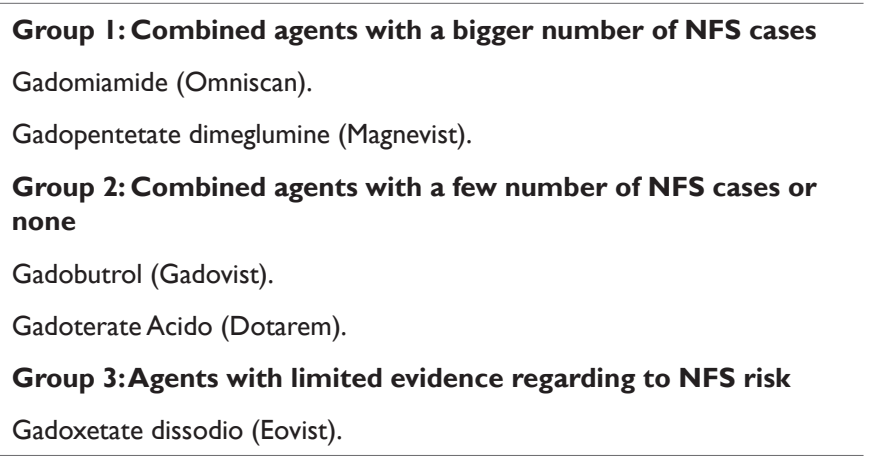

\section{Conclusion}

Besides its frequent use for diagnosing and treating, iodinate contrast may in addition to cause CIN accelerate renal dysfunction and need to RRS and increase treatment costs. Prevention strategies such as hydration with saline solution is important because it reduces further complications. Regarding to the use of gadolinium as a contrast substance the worst effect may happen to those with previous renal dysfunction who use linear molecules. Because it is related to an increased risk of NSF it is recommended to dialyze these patients right after they are exposed to gadolinium.

\section{Acknowledgements}

None.

\section{Conflict of interest}

Author declares that there is no conflict of interest.

\section{References}

1.https://www.acr.org/-/media/ACR/Files/Clinical-Resources/Contrast_ Media.pdf

2. Lima FV, Gruberg L. Here we go again: Trying to prevent contrast induced nephropathy. Cardiovasc Revasc Med. 2017;18(5):313-314.

3. KDIGO Clinical Pratice Guideline for Acute Kidney Injury. Official Journal of the International Society of Nephrology. 2012;2:1-141.
4. KDIGO 2012 Clinical practice guideline for the evaluation and management of chronic kidney disease. Kidney International Supplements. 2012;3(1):1-136.

5. Laranja SMR, Dande CV. Lesão Renal Aguda e Doença Renal Cronica Agudizada: epidemiologia, evolução e prevenção. Atualidades em Nefrologia. Ed Sarvier; 2012. p. 420-425.

6. Vanommeslaeghe F, de Mulder F, de Bruaene CV, et al. Selecting a strategy for prevention of contrast-induced nephropathy in clinical practice: an evaluation of different clinical practice guidelines using the AGREE tool. Nephrol Dial Transplant. 2015;30(8):1300-1306.

7. Rudnick MR, Berns JS, Cohen RM, et al. Nephrotoxic risks of renal angiography: contrast media-associated nephrotoxicity and atheroembolism-a critical review. Am J Kidney Dis. 1994;24(4):713-727.

8. Weisbord SD, Gallagher M, Jneid H, et al. Outcomes after Angiography with Sodium Bicarbonate and Acetylcysteine. NEJM. 2017.

9. Mehran R, Aymong ED, Nikolsky E, et al. A simple risk score for prediction of contrast-induced nephropathy after percutaneous coronary intervention: development and initial validation. $\mathrm{J} \mathrm{Am} \mathrm{Coll} \mathrm{Cardiol.}$ 2004;44(7):1393-1399.

10. Pannu N, Wiebe N, Tonelli M. Prophylaxis strategies for contrast-induced nephropathy. JAMA. 2006;295(23):2765-2779.

11. Tepel M, van der Giet M, Schwarzfeld C, et al. Prevention of radiographic contrast agent induced reductions in renal function by acetylcysteine. N Engl J Med. 2000;343(3):180-184.

12. Trivedi H, Daram S, Szabo A, et al. High-dose N-acetylcysteine for the prevention of contrast-induced nephropathy. Am JMed. 2009;122(9):874.

13. Tarek A Ibrahim, Ramzy H El-Mawardy, Ahmed S El-Serafy, et al. Trimetazidine in the prevention of contrast-induced nephropathy in chronic kidney disease. Cardiovasc Revasc Med. 2017;18(5):315-319.

14. Daftari Besheli L, Aran S, Shaqdan K, et al. Current status of nephrogenic systemic fibrosis. Clin Radiol. 2014;69(7):661-668.

15. Abu-Alfa AK. Nephrogenic systemic fibrosis and gadolinium-based contrast agents. Adv Chronic Kidney Dis. 2011;18(3):188-198.

16. Jerry Yee. Prophylactic Hemodialysis for protection against gadolinium-induced nephrogenic systemic fibrosis: a doll's house. Adv Chronic Kidney Dis. 2017;24(3):133-135. 